\title{
LÉGISLATION
}

\section{La Taxe de mainmorte et les Entreprises concédées - Principes de la taxation et de l'éxonération - Tarifs}

Par Paul Bougault, Avocal d̀ la Cour d'Appel de Lyon.

\begin{abstract}
Il est certain que, malgré les traités consacrés à la législation fiscale et de nombreux articles parus dans la presse technique, quelques concessionnaires d'entreprises, soit hydrauliques, soit électriques, ignorent encore dans quels cas ils sont exempts de la taxe dite de la "mainmorte "et dans quels cas ils ne peuvent se refuser à la payer. Plusieurs s'ćlonnent de la devoir au fisc; d'autres, sans la discuter, voudraient la faire payer à la commune dont ils sont concessionnaires; peut-être même, évipemment en plus petil nombre, en existe-t-il qui s'étonnent de ne pas se la voir réclamer. Un rapide exposé des règles générales parait nécessaire.
\end{abstract}

Origine de la mainmorte. - Sa nature. - La taxe de mainmorte est exigée par l'Etat, sur les immeubles appartenant à certaines collectivités, pour remplacer les droits de mutation qu'il toucherait souvent si ces immeubles appartenaient à des particuliers. La loi du 20 février 1849 a donné une énumération de ces collectivités : les départements, les communes, les sociétés anonymes. D'une façon plus générale, la loi du 31 mars 1903 (D. P., 1904-4-17) dans son article 2 , a stipulé : "La taxe annuelle représentative des divers droits de transmission entre vifs, établie par la loi du 20 févier 1849 est due par toutes les collectivités qui ont une existence propre et qui subsistent indépendamment des mutations qui peuvent se produire dans leur personnel, d̀ l'exception des sociétés en nom collectif et des sociétés en commandite simple. "Cette dernière phrase signifie que les " sociètés de personnes " par opposition à "sociétés de capitaux " bénéficient d'une exemption ; par exemple, la société en commandite simple ne paiera pas la taxe, alors que la société en commandite par actions y sera soumise.

La loi de 1903, si l'on se reporte aux travaux préparatoires, a été considérée par l'Administration comme nécessaire, pour élargir le cadre de sa devancière, la loi du 20 février 1849 que la jurisprudence administrative se refusait à interpréter comme englobant, par assimilation, des collectivités qui n'y étaient pas expressément désignées. Echappaient à la taxe avant la loi de 1903 : les sociétés en commandite par actions, les sociétés à responsabilité limitée fondées avant la loi du 24 juillet 1867, les sociétés civiles par actions n'ayant pas le caractère parfait de l'anonymat.

Sur le sens très restrictif de la jurisprudence, on peut consulter les décisions suivantes : 9 mai 1873 (D. P. 75.3.15, aff. Société des Raffineries de la Méditerranée); dans cette instance, le Conseil d'Etat a statué à l'égard de ce que l'on appelait alors une société à responsabilité limitée : créé avant 1867, la Société des Raffineries n'avait pu se constituer qu'avec l'autorisation du Gouvernement, et n'avait pas sollicité sa modification en une société anonyme, conforme à la loi nouvelle, comme elle aurait pu le faire, en usant de l'article 47 de cette loi : "Les sociétés à res" ponsabilité limitée pourront se convertir en sociétés anonymes " dans les termes de la présente loi, en se conformant aux condi" tions stipulées pour la modification de leurs statuts. " L'arrèt du 26 octobre 1894 (D. P. 95.5.163, aff. Société des QuaranteSols) : le Conseil d'Etat a déclaré que le fait qu'une partie des titres de la société civile appartient à une société anonyme n'en modifie pas le caractère et ne la soumet pas à la taxe de la mainmorte. Les deux arrêts cités dans Dalloz 1900.3.1. : l'un du 25 novembre 1898, dans lequel la Société des Moulins du Bazacle a été maintenue comme imposée à la taxe, parce que, tout en se. déclarant Société civile anonyme, elle avait, d'après ses statuts, tous les caractères d'une société vraiment anonyme. L'autre du 23 décembre 1898 dans lequel, au contraire, le Conseil affranchit de la taxe la Société civile de la rue Ville-ès-Martin, à SaintNazaire, parce qu'elle ne correspond pas vraiment à la société anonyme. Il semble, d'après le rapprochement dess deux arrèts que, pour le Conseil d'Etat, le critérium de la société anonyme réside dans le fait de la limitation de l'engagement de l'actionnaire à sa mise (1). En ce sens, on peut trouver un précédent dans un arrêt du 23 juillet 1892 (D. P. 1892.5.169, aff. Société immobilière Léopold) dont le sommaire est ainsi conçu : "Une "Société ne peut contester qu'elle soit anonyme et, par suite, " passible de la taxe de mainmorte, Iorsque ce caractère qui " ressort de l'ensemble des pièces du dossier, est encore accentué

(1) S'il en est ainsi, on peut se demander arec une certaine anxiété, quelle décision sera prise par l'Administration des Contributions directes au sujet des sociétés à responsabilité limitée créées par la loi du 7 mars 1925 . Ces sociétés se rapprochent évidemment de la société anonyme, notamment par la possibilité de limiter la responsabilité pécuniaire au montant de l'apport de l'actionnaire; elles se rapprochent aussi de la société de personnes, par une série de clauses: la possibilité de se constituer, sans faire intervenir le minimum de 7 membres (art. 5 de la loi du 7 mars 1925); les formalités nécessaires pour la cession des parts sociales (art. 23). Si le premier élément (limitation de la responsabilité) est primordial, on peut redouter l'application de la mainmorte à l'immeuble. 
" par les dispositions des statuts qui limitent la responsabilite " des actionnaires au montant de leur apport et, par un article "les statuts qui corrobore ces dispositions en exigeant que les "administrateurs rappellent cetle clause de limitation dans les " différents contrats qu ils passeront."

Comme on l'a fort bien dit, la laxe de mainmorte a ćlé inspirée à l'Etat par un esprit de.... vengeance : il ne pardonne point aux sociétés par actions, aux communes, aux départements, de n'aliéner leurs immeubles que très rarement, et surtout de ne pas mourir à l'instar d'une persomne physique. Il les frappe d'un impòt spécial en raison de la fixité, de la quasi-perpétuité de leur propriété. Il est donc naturel de dire, comme conséquence, que la taxe de "maimmorte " est un accessoire de limpòt foncier, car ce dernier impôt étant toujours à la charge du propriétaire réel, il en résultera fatalement que son accessoire sera également réclamé à ce propriétaire et non au locataire ou au détenteur à titre précaire de limmeuble. Nous allons trouver immédiatement une application de ce principe dans la matière de distributions d'énergie.

Cas général en matière de concession municipale. - Supposons une société anonyme, concessionnaire de l'éclairage d'une commune; supposons encore que cette concession comprenne une usine de production (gaz ou courant électrique); enfin, précisons bien que le cahier des charges est muel sur la question du paiement des impôts dus à l'Etat. Nous nous trouvons en présence de deux collectivités qui, par leur nature juridique, sont susceptibles de se voir réclamer la laxe de mainmorte. Lá jurisprudence et la doctrine sont formelles sur ce point. La collectivité qui sera débitrice de l'impôt, c'est la commune et non pas la société concessionnaire, car c'est la commune seule qui devient propriétaire, dès leur construction, des ouvrages que le concessionnaire établit pour faire son exploitation. Cette responsabilité de la commune devant l'impôt à la décharge du concessionnaire, a été mise en vive lumière, tant pour l'impôt foncier que pour la mainmorte dans l'affaire jugée par le Conseil d'Etat le 31 mars 1922 (voir le texte dans mon "Nanuel pratique de la Contribution foncière " page 104 en note). Quelques personnes ont voulu voir, dans cette décision, une innovation heureuse et originale. Il est facile de se rendre compte que c'est au contraire la reproduction d'une jurisprudence très ancienne et applicable à toute concession municipale (consulter sur cette précision et les arrêts qui vont suivre Dalloz, Code des Lois pol et adm. annoté, Vo Contributions directes, Nos 9517 et suivants).

Par exemple, le Conseil d'Etat, le 28 février 1890 (D. P. 1891 1891.5.141, aff. Compagnie nouvelle d'Eclairage par le gaz) a proclamé qu'une société anonyme à qui une ville a concédé l'établissement et l'exploitation d'une usine à gaz, qui a construit cette usine sur un terrain appartenant à la commune, et qui, à l'expiration de la concession doit remettre à la commune l'usine et ses dépendances, sans indemnité et en bon état de fonctionnement, ne peut ètre considérée comme propriétaire du dit établissement, et, par suite, n'est pas imposable à la mainmorte.

Cas particulier d'une distribution municipale d'énergie en conformité du cahier type.- Les rédacteurs du cahier des charges type n'ont pas oublié ces principes : ils ont compris, facilement, que, s'ils n'écrivaient pas, au sujet des impôts, une stipulation formelle, les communes en deviendraient débitrices vis-à-vis du trésor, puisqu'en raison de l'article 22, elles reçoivent gratuitement de leur concessionnaire les ouvrages que celui-ci aura placés : elles sont donc réellement propriétaires. Seule, la jouissance de ces ouvrages est différée pour elles jusqu'à l'expiration du contrat : Telle est l'origine de eet article bien comm (article 29) dont le premier alinéa est si lavorable aux communes : "Tous " les impóts établis par l'Llat, le département ou la commune, " y compris les impôts relatifs aux immeubles, de la distribution, "seront à la charge du concessionnaire." Il est évident que si la colation était faite au nom de la commune, celle-ci forte de son droit contractuel, serail armée pour en réclamer la restilution au concessionnaire et entreprendre la procédure de mulation de cote (voir sur celle procédure le Mantel précité de la Contribulion foncière, page 106) (1). Les concessions de distribulion d'éclairage englobant une usine de production sont rares; aussi l'impôt foncier et, par conséquent, le droit de mainmorte, n'est pas souvent appliqué en ce qui concerne les dépendances de la concession : c'est pour ce molif que nous n'avons aucune jurisprudence à ciler.

Différence au point de vue de la mainmorte entre la concession hydraulique et la concession municipale de distribution d'énergie.En maticre de concession hydraulique, donnée en application de la loi du 16 oclobre 1919, la situation au point de vue de la laxe de mainmorte est toute différente.

()n ne saurait trop rappeler que l'aulorite concédante est l'Etal, par conséquent que l'Elat est le véritable propriétaire qui, à l'expiration du contrat, reprendra la libre jouissance de Loutes les dépendances de la concession. Il apparaîtrait done, comme absurde au premier chef que l'Etat perçul un impot destiné it compenser l'absence de mutation d'un bien qui devient sa propriété, el la meilleure des compensations sera la jouissance complète, dans l'avenir, de cette usine hydroélectrique que le concessionnaire établit, sans que l'autorité concédante ait à dépenser la moindre somme. Aussi, il importe peu que le cahier des charges contienne un article conçu dans les mèmes termes que l'arlicle inséré dans le cahier des distributions électriques, savoir : article 53 : "Tous les impôts élablis ou à établir par l'Etal, les départements ou les communes, seront à la charge du concessionnaire. "Cela est vrai pour l'impôt foncier; car la nature même de cet impôt et son origine ne constituent pas un obstacle au paiement par le concessionnaire ; ce serait impossible pour la mainmorte que nous avons défini ci-dessus : un impôt de compensation exigé pour des immeubles dont certaines collectivités citées par les lois de 1849 et de 1903 sont propriétaires ; el l'Etat n'est pas compris parmi ces collectivités citées.

Si l'on ne peut poinl invoquer de jurisprudence pour les concessions hydrauliques, parce qu'elles sont encore trop récentes pour avoir causé des procès, on peut trouver de nombreuses décisions du Conseil d'Etat relativement aux Compagnies de chemins de fer qui donnent bien le type de la concession

(1) Il ne faudrait pas croire que l'arrêt du Conseil d'Etat du 9 novembre 1895 (Dalloz 1896.3.88, aff. ville de Paris) soit opposí à la thèse ci-dessus bien qu'il porte dans son sommaire une phrase que l'on pourrait mal interpréter : "Lorsqu'aux termes du traite " par lequel la Ville a concédé à un industriel des constructions " élevées sur une promenade publique, ces constructions doivent "faire retour à la Ville sans indemnité aucune, et que le conces" sionnaire doit acquitter lons les impôts y compris la mainmorte, " la Ville doit être considéréc comme propriétaire de ces construc" tions et est, par suite, passible de la taxe de mainmorte. "I résulte, en effet, comme le remarque une note parue au Dalloz, que l'Administration des contributions directes a parfaitement le Iroit de considérer comme tenue de l'impôt la ville cle Paris, puisque celle-ci est propriétaire des ouvrages; mais, si la ville de Paris le clésire, rien ne lui interdit de s'exonérer, en faisant usage de son contrat à l'égard du concessionnaire. 
octroyée par l'lital avec retour forméllement assuré par l'autorité concédante.

Et comme l'explique très bien le Code des lois pol. et adm. de Dalloz, Vo Contributions directes, No 9519 : "La jurisprudence " est fondée sur ce que les terrains occupés par les chemins et " canaux, bien que soumis à la contribution foncière en vertu du " cahier des charges (1) lont partie du domaine public et n'appar" tiemnent par conséquent pas aux Compagnies dans le sens de " la loi du 20 février 1849. " Dès le lendemain de l'apparition de la loi, le Commissaire du gouvernement Cornudet, établissait dans ses sonclusions (arrếl du 8 février 1851, D. P. 51.3.49, aff. Compagnie des chemins de fer du Centre) que les dépendances immobilières de la concession n'élaient pas pour la Compagnie une vraie propriété, ct l'arrêt en se basant sur les termes de la loi donne une solution très intéressante : "Considérant, dit-il, " que la laxe représentative des droits de transmission entre "vifs el par décés, qui a elé créé par la loi du 20 février 1849 " ne porte que sur les immeubles passibles de la contribution "foncière qui appartiennent aux établissements ou personnes " "ivils désignés par l'article $1^{\text {er }}$ de cette loi. "Or, les établissements ou personnes cités sont: les départements, communes,

(1) Le cahier des charges des chemins de fer d'intérêt général porte un article 62 (voir code des Lois Pol. an. Vo Voirie par chemins de fer et tramways) : "La contribution foncière sera " établie en raison de la surface des terrains occupés par le chemin " de fer et ses dépendances" la cote en sera calculée comme les " canaux, conformément à la loi du 25 avril 1803 . Les bâtiments " ct magasins dépendant de l'exploitation du chemin de fer seront " assimilés aux propriétés bàties de la localité. 'Toutes les contri"butions auxquelles ces édifices pourront être soumis, seront "aussi bien que la contribution foncière, à la charge de la Compagnie. "En ce qui concerne l'impôt foncier, remarquons que même les chemins de fer de l'Etat doivent le payer, car on ne sanrait oublier l'article 9 de la loi du 22 décembre 1878, D. P. 79.4.12 : "Les chemins de fer exploités par l'Etat sont soumis en ce qui " concerne les droits, taxes et contributions de toute nature au "même régime que les chemins de fer concédés. " Cet article qui, ainsi que l'indiquent les travaux préparatoires de la loi, avait pour but de supprimer les droits d'enregistrement que l'exploitation des chemins de fer de l'Etat payait pour les marchés passés par elle a eu pour effet, en sens contraire, de la sommettre aux impôts directs que les compagnies supportent. les sociétés, les établissements publics; on ne rencontre pas Je nom de l'Etat. La même jurisprudence se trouve encore dans un arrêt de même date, en ce qui concerne les canaux de la Compagnie du Loing.

Enfin, on peut encore considérer comme très instructive la lecture des arrêts suivants : l'arrêt du 22 août 1853 (D. P. 54.3.76, aff. Compagnie des chemins de fer de Paris à Orléans) dans lequel le Conseil d'Etat affirme que les locaux affectés dans les gares du cheminde fer au service du buffet, sont exempts de la taxe de mainmorte, comme faisant partie des dépendances du domaine concédé : l'arrêt du 11 janvier 1866 (D. P. 66.3.72, Compagnie des chemins de fer de Lyon) les maisons sítuées dans les dépendances de la gare de Mâcon sont exemptes au même titre. Doivent au contraire, être soumis à la taxe, les immeubles qui, bien qu'appartenant à la Compagnie, sont situés en dehors du domaine public (arrêt du 6 janvier 1853, D. P. 1854.3.1).

Tarif. - D'une façon générale, pour ceux de nos lecteurs qui seraient intéressés par la question de la mainmorte, nous donnerons les indications suivantes, au sujet des tarifs.

Aujourd'hui et jusqu'au 1er janvier 1927, nous sommes encore sous l'empire du tarif édicté par l'article 5 de la loi du 31 juillet 1920 : le droit est calculé en raison de 130 centimes par franc du principal de la contribution foncière; il est applicable aux immeubles des sociétés. Les départements, les communes, et certaines sociétés particulièrement intéressantes bénéficient d'un tarif de faveur de 85 centimes. On sait qu'aujourd'hui encore, le principal de la contribution foncière est donné par le $10 \%$ du revenu net.

Mais, en raison de l'article 26 de la loi du 3 août 1926, les impôts à partir du 1er janvier 1927 vont être majorés. Le taux de la contribution foncière, notamment en vertu de l'article 23 de la même loi, va ètre de $18 \%$. Multiplier le pourcentage trouvé au moyen de ce taux, par 130 ou 85 centimes, c'eût èté grever la propriété mainmortable d'une surtaxe formidable. Aussi, le coefficient de la mainmorte a-t-il été abaissé, et sera, à partir

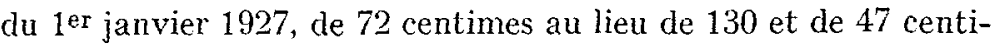
mes au lieu de 85 . (Voir sur ce point l'article 24 de la loi du 3 août 1926). 